\title{
17 Gestaltungselemente und Usability
}

Katja Derr, Edith Mechelke-Schwede

Bei der Gestaltung digitaler (Lern-)Umgebungen spielt die Nutzbarkeit oder Usability eine entscheidende Rolle. Das Design der Oberfläche (des Frontend) ermöglicht es Nutzer*innen, eine Software zu bedienen oder auf einer Website zu navigieren. In ihrer optischen Gestaltung spiegelt sich die interne Struktur einer Software oder Website, und eine geringe Usability wird zu Recht den Eindruck vermitteln, dass ein Programm konzeptionell nicht durchdacht ist.

Schlechte Bedienbarkeit und daraus resultierender Orientierungsverlust kann schnell zu Frustration führen - und in webbasierten Lernumgebungen zum Abbruch der Lernhandlungen (Duckwitz und Leuenhagen 2004). Wenn Lernende wichtige Informationen nicht finden, oder eine „Betriebsanleitung“ benötigen, um sich zurechtzufinden, wirkt sich das erheblich auf die Akzeptanz der Lernplattform insgesamt aus. Evaluationsanalysen haben hohe Korrelationen zwischen der Zustimmung zur Usability und der Zustimmung zum gesamten Projekt gezeigt, dies gilt für Lernsoftware (Granić und Ćukušić 2011) ebenso wie für professionelle Unternehmenssoftware (Scholtz, Mahmud und Ramayah 2016).

Laut DIN-Norm ISO 9241-11 bezeichnet Usability ,das Ausmaß, in dem ein Produkt durch bestimmte Nutzer in einem bestimmten Nutzungskontext genutzt werden kann, um bestimmte Ziele effektiv, effizient und zufriedenstellend zu erreichen". In der Literatur wird mittlerweile häufiger von User Experience gesprochen, um das komplexe Zusammenspiel zwischen Nutzer*in, Inhalt und digitaler Umgebung zu beschreiben (z. B. Marcus 2013). In diesem Kapitel wird der Fokus vor allem auf den Gestaltungselementen liegen, die zur besseren Orientierung im optes-Vorkurs beitragen.

Da der Nutzungskontext von Websites sich stark unterscheidet, wird Usability immer zweckbezogen betrachtet (Gizycki 2002, S. 2). So wird es auf Unterhaltungsseiten meist akzeptiert, dass viele Bildelemente die Aufmerksamkeit auf sich ziehen. In webbasierten Lernumgebungen hingegen werden Ablenkungen und Abschweifungen als störend empfunden. Nielsen konnte zeigen, dass Jugendliche und junge Erwachsene durchaus zwischen Internet- und Computernutzung in der Freizeit und im Schul- bzw. Hochschulkontext unterscheiden. Mit zunehmendem Alter der Lernenden sank außerdem die Akzeptanz von unruhigen und unübersichtlichen Websites. Spätestens von einer universitären Lernumgebung erwarten Lernende sachliche und übersichtlich aufbereitete Informationen (Nielsen 2010).

Natürlich ist die Beobachtung, dass sich Gestaltung auf (E-)Lernprozesse auswirken kann, nicht neu. Nielsens Usability Prinzipien (1994) oder die Vorschläge für didaktische Designs von Reeves (1994) stammen aus den 1990er Jahren und 
werden auch 20 Jahre später noch zitiert (z. B. Clark und Mayer 2011; Lera et al. 2013). Ein Problem ist, dass die Umsetzung dieser Regeln angesichts der ständigen technischen Weiterentwicklungen permanent überprüft und angepasst werden muss. Lernumgebungen wie ILIAS werden stetig erweitert und immer mächtiger und damit komplexer.

Auch im optes-Projekt wurden solche Features eingesetzt, beispielsweise der LoK (siehe Kapitel 14). Andere wurden zusätzlich entwickelt bzw. extern beauftragt, wie die Fähigkeitsmatrix (Kapitel 6) oder ein Plug-in für Adaptive Trainings (Kapitel 8). Auch für die Einbindung mathematischer Lerninhalte war es notwendig, zusätzliche Plug-ins entwickeln zu lassen (siehe Kapitel 10 und Kapitel 15). Dementsprechend groß ist die Herausforderung, die umfangreichen Lernmaterialien intuitiv und niederschwellig nutzbar zu machen.

Generell wurde im optes-Projekt im Sinne der besseren Disseminierbarkeit an andere Hochschulen darauf geachtet, dass alle Materialien und Plug-ins auf einer Standard-Installation installiert werden können. Das gilt auch für das Erscheinungsbild: Die importierten Lernmaterialien lassen sich mit dem schon bestehenden Design einer ILIAS-Installation zusammenführen.

Für die Lernmodule wurde ein Style entwickelt, der nach Import der optesLernmodule aktiviert werden muss (Lernmodule: Standard). So ist die saubere Darstellung der Texte, Definitionen und Formeln gewährleistet. Darüber hinaus wurden diverse Icons für die Gestaltung der optes-Lernmaterialien entwickelt. Sie werden automatisch mitimportiert (ab ILIAS 5.4) und können dann farblich an den jeweiligen Hochschul-Style angepasst werden.

\subsection{Einstieg}

Die optes-Materialien werden an verschiedenen Hochschulen in unterschiedlichen Vorkurs-Szenarien eingebunden. Dementsprechend unterschiedlich sind die Einstiegs- und Informationsseiten gestaltet. Es bietet sich aber natürlich an, eine Übersicht über den Ablauf und über die Lerninhalte des Kurses zu geben. Abbildung 1 und 2 zeigen beispielhaft den Einstieg in die Studienvorbereitung Mathematik an der DHBW Mannheim. ${ }^{1}$

Neben allgemeinen Informationen zu Ablauf und Kursterminen wird im offenen Bereich der ILIAS-Plattform auch über den Sinn und Zweck des Vorkurses informiert. Durch Klick auf „Warum ein Mathematik-Vorkurs?“ können sich angehende Studierende über die Rolle der Grundlagemathematik für ihr Studium informieren. Hier werden auch Auswertungen von Evaluationen früherer Jahrgänge sowie Befragungen von Studierenden zusammengefasst, um schon mal einen ersten Bezug zum Studium herzustellen.

\footnotetext{
${ }^{1} \mathrm{https}: / /$ studienstart.dhbw-mannheim.de [10.06.2020].
} 


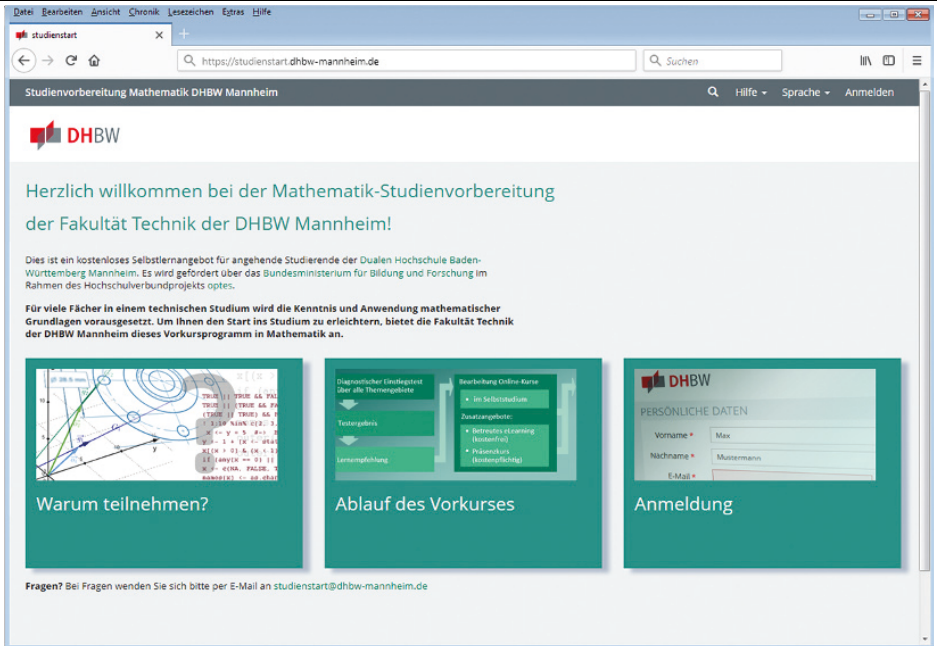

Abbildung 1: Einstieg: Übersichtsseiten DHBW Mannheim

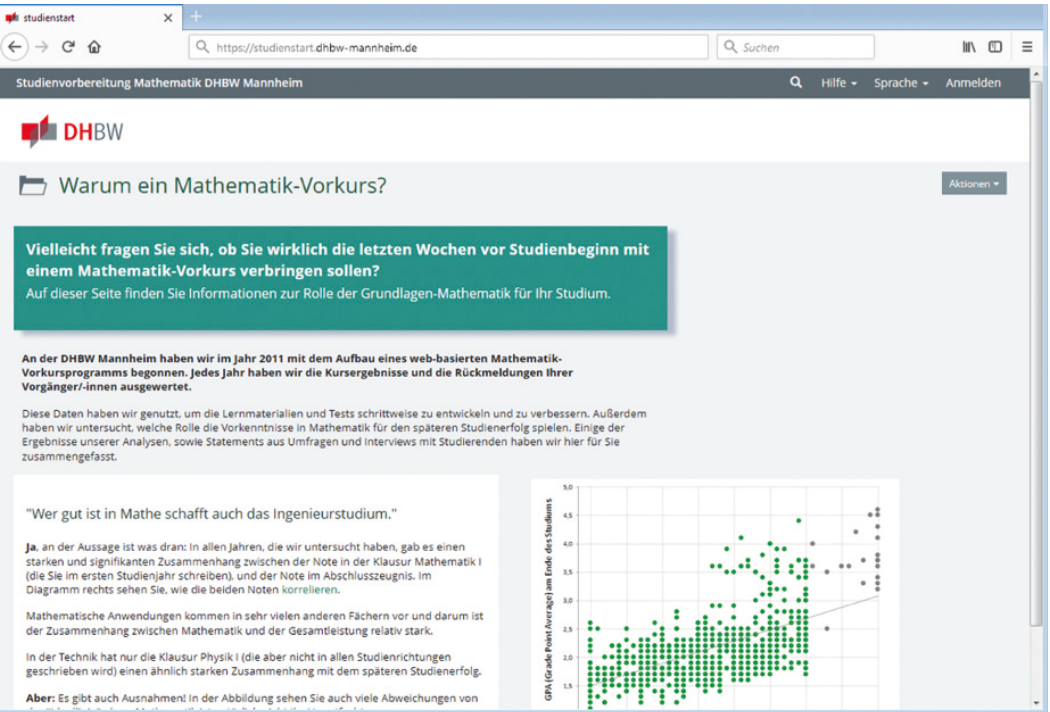

Abbildung 2: Einstieg: „Warum ein Mathematik-Vorkurs?“ 


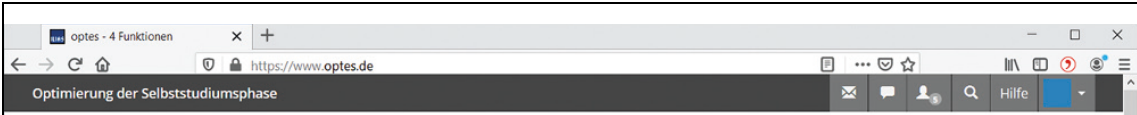

\section{optes}

MEIN OPTES - EINSTIEG IN OPTES - ZULETZT BESUCHT -

$f(x) 4$ Funktionen

Themen: Elgenschaften von Funktionen | Rationale Funktionen | Umkehrfuniktionen | Exponentlat- und Logarithmusfuniktionen

Willkommen im Kurs "4 Funktionen"

Kursinformationen finden Sie hier

Dieser Kurs dient zur Auffrischung Ihrer Kenntnisse über das Erstellen und Arbeiten mit Funktionen.

Sollten Sie in diesem Lernmodul auf mathematische Ausdrücke / Formulierungen treffen, die Ihnen unbekannt sind, empfehlen wir Ihnen die Bearbeitung des Kurses " 0 Mathematische Grundlagen"

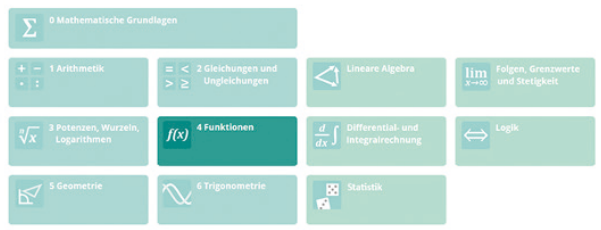

Der Kurs "4 Funktionen" ist für eine Bearbeitungszeit von 10 bis 12 Stunden ausgelegt. Dies ist allerdings nur ein Richtwert. Je nach Vorwissen und individuellem Tempo kann es natürlich sein, dass Sie mehr oder deutlich weniger Zeit benötigen. Ein entsprechender Zeitplan kann Ihnen zur Planung ihrer individuellen Lernzeit behilflich sein.)

Abbildung 3: Einstieg in den Lernzielorientierten Kurs: Kursinformationen LoK Funktionen

Im Sinne eines Advance Organizer (Krause und Stark 2006) enthält auch der Einstieg in jeden Lernzielorientierten Kurs (LoK) eine Informationsseite, die den Ablauf und die Lernziele des Kurses beschreibt. Sie kann über ein aufklappbares Akkordeon jederzeit abgerufen oder ,zusammengeklappt“" werden (Abbildung 3). Auf der Einstiegsseite des Kurses werden außerdem die Lernmaterialien des Kurses nach Lernzielen geordnet dargestellt (siehe auch Kapitel 14).

Nach Klick auf das zum Lernziel gehörige Lernmodul kommen die Lernenden auf die Einstiegsseite des Lernmoduls. Eine repräsentative Abbildung und ein kurzer Einleitungstext zeigen auf, worum es im Lernmodul geht. In einem grafisch abgesetzten Kasten werden die Kenntnisse und Fertigkeiten aufgelistet, die die Lernenden nach Bearbeitung des Lernmoduls erworben haben. Abbildung 4 zeigt die Einstiegsseite des Lernmoduls Stereometrie im LoK Geometrie. 


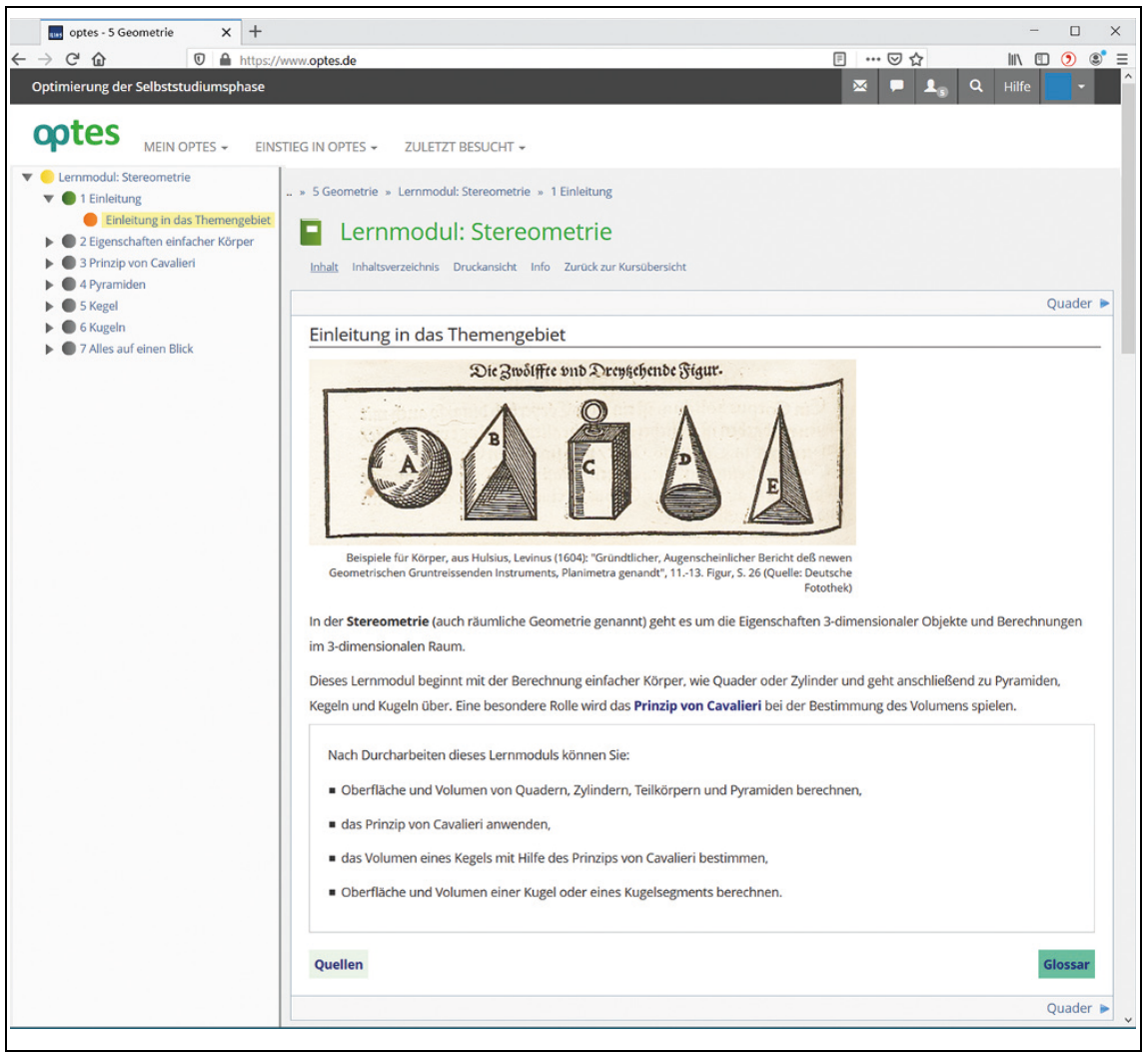

Abbildung 4: Einstiegsseite Lernmodul: Einleitung in das Themengebiet Stereometrie (LoK Geometrie)

\subsection{Navigation und Orientierung}

Das didaktische Konzept von optes beinhaltet eine Nutzerführung über Diagnostische Tests und darauf basierende Lernempfehlungen. Es ist aber durchaus denkbar, dass einmal zusätzliche Inhalte nachgeschlagen werden müssen oder dass Teilnehmer*innen Themengebiete bearbeiten möchten, für die sie keine Lernempfehlung erhalten haben. Darum sollte es jederzeit möglich sein, sich einen Überblick über die Lerninhalte zu verschaffen und zwischen den Materialien zu navigieren. Damit das ohne Orientierungsverluste gelingen kann, sind alle LoKs und in den LoKs alle Lernmodule nach dem gleichen Schema aufgebaut. Über eine intuitive Nutzerführung wissen die Lernenden jederzeit, wo sie sich gerade im Lernmodul befinden. 
Abbildung 5 zeigt den schematischen Aufbau einer typischen Lernmodulseite. Über die Breadcrumb-Navigation können die Lernenden jederzeit zur Übersichtsseite des Lernzielorientierten Kurses zurückkehren. Über Pfeiltasten über bzw. unter dem Text kann zur nächsten/vorherigen Seite geblättert werden.

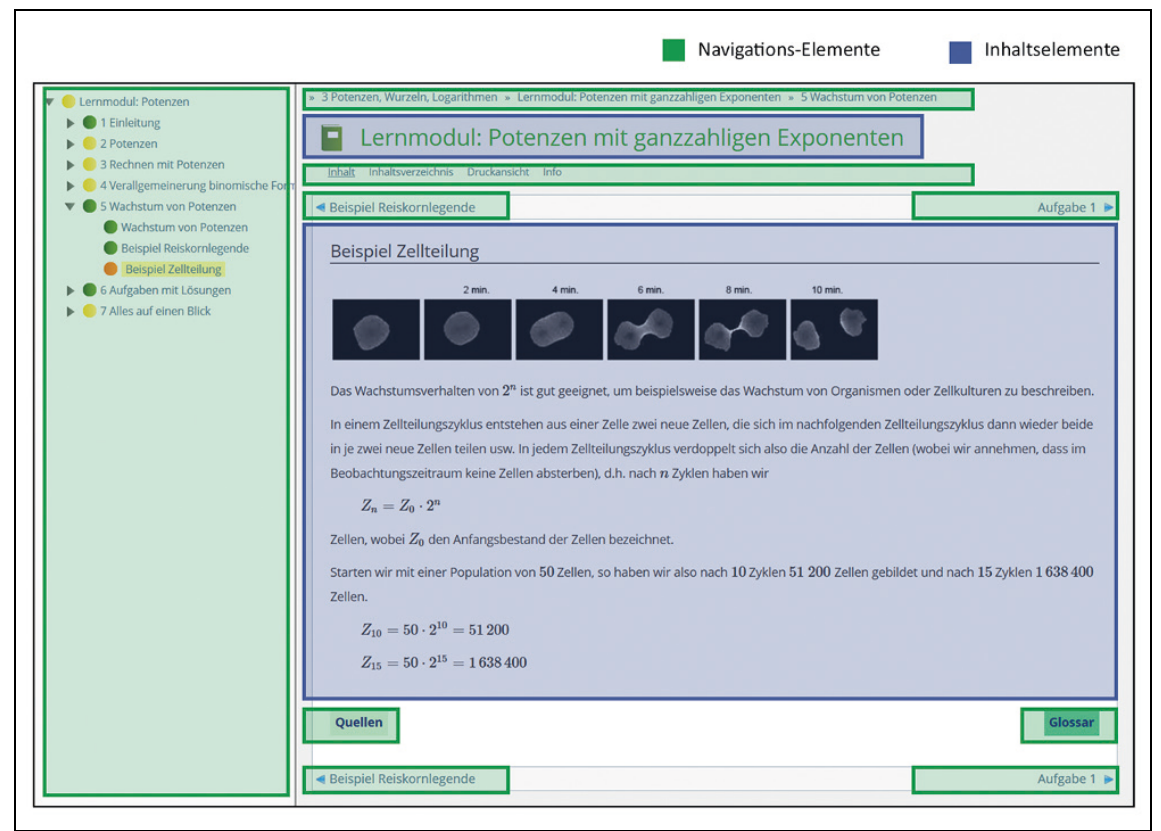

Abbildung 5: Aufbau einer Lernmodulseite: Navigations- und Inhaltselemente

Die Kapitel, Unterkapitel und Seiten sind über die Inhaltsübersicht jederzeit erreichbar. Die Kapitel lassen sich auf- und zuklappen. Damit die Lernenden sehen können, welche Seiten sie schon bearbeitet haben, wird in der Inhaltsübersicht über farbige Auszeichnungen der Lernfortschritt angezeigt (siehe Tabelle 1).

Tabelle 1: Übersicht über Icons der Lernfortschrittsanzeige

bearbeitet

in Bearbeitung

wird aktuell bearbeitet

noch nicht begonnen

nicht erfolgreich bearbeitet 
Die Lerninhalte werden nach dem Einstieg in das Themengebiet analog zu einem Lehrbuch aufeinander aufbauend erläutert. Inhalte oder Begriffe, die im Lernmodul nicht explizit erläutert oder vorausgesetzt werden, sind mit dem Glossar verlinkt. Falls darüber hinaus ein Begriff nicht bekannt ist, kann über den Button „Glossar“ jederzeit der Link auf das gesamte mathematische Glossar geöffnet werden. Dieser Button befindet sich auf jeder Lernmodulseite rechts unten.

Am Ende des Lernmoduls erfolgt auf der Seite „Alles auf einen Blick“ eine Übersicht über die Kernthemen, die im Lernmodul behandelt wurden (Abbildung 6). Auf der letzten Seite jedes Lernmoduls („Zusammenfassung“, siehe Abbildung 7) werden die Lernziele von der Einstiegseite nochmals wiederholt. Beide Seiten dienen zur Rekapitulation und zur Vergegenwärtigung, was gelernt (oder noch nicht gelernt) wurde.

Auf der letzten Lernmodulseite wird außerdem auf das zum Lernmodul gehörige Training verwiesen. Mit der Bearbeitung des Trainings wird im Sinne einer Selbstevaluation bzw. Monitoring (Winne 2004) der Lernprozess für dieses Lernziel abgeschlossen.

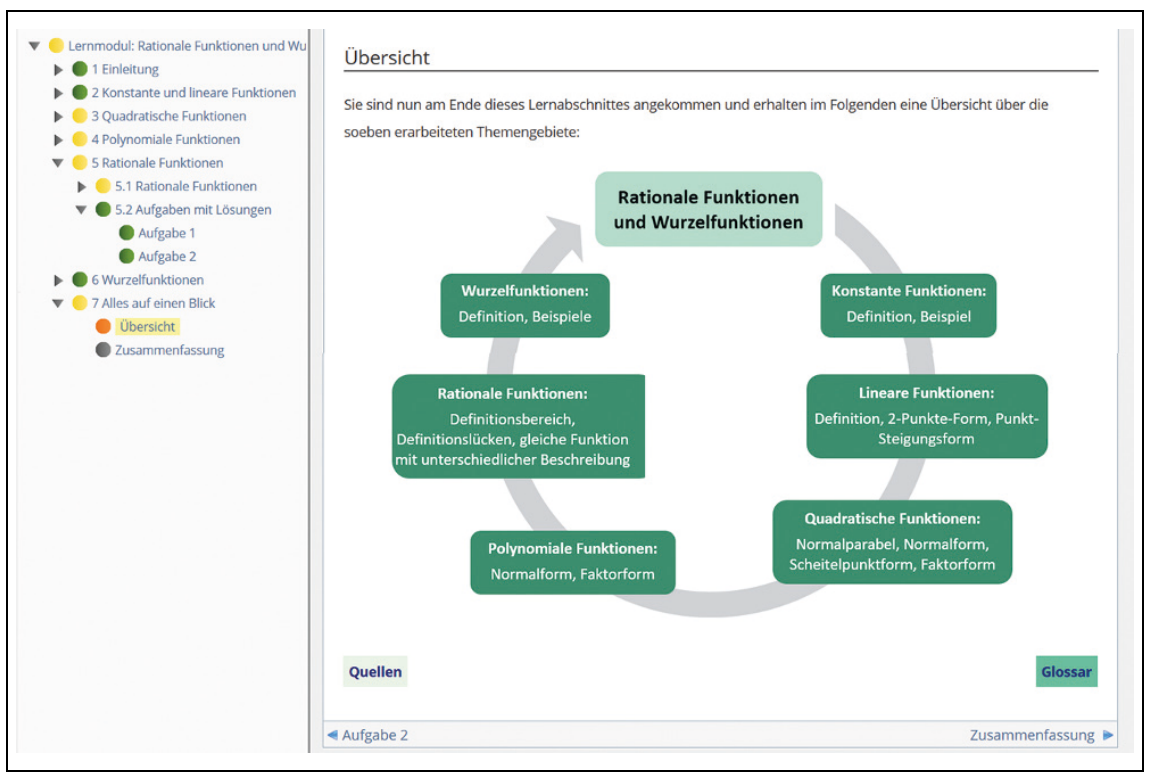

Abbildung 6: Screenshot Lernmodul Rationale und Wurzelfunktionen - Übersicht 


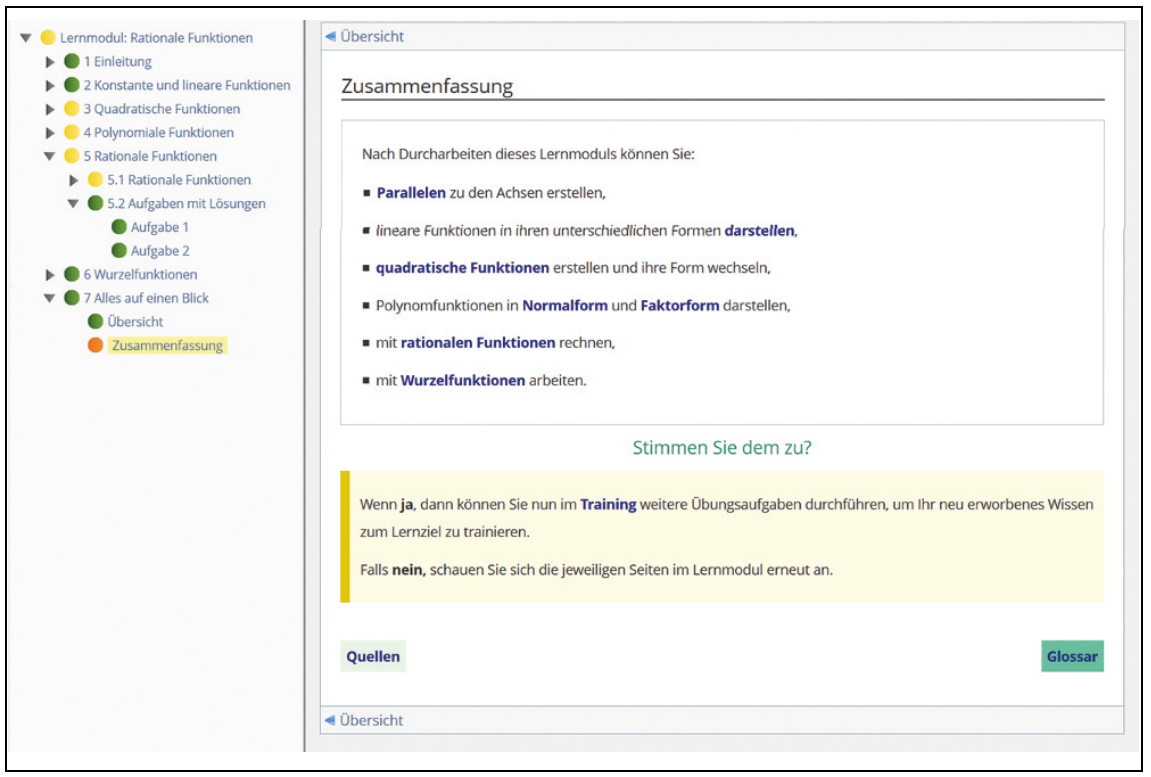

Abbildung 7: Screenshot Lernmodul Rationale und Wurzelfunktionen - Zusammenfassung

\subsection{Grafische Elemente und Icons}

Normaler Fließtext in optes-Lernmodulen oder -Aufgaben wird als Standard-Text schwarz auf weißem Hintergrund gesetzt. Alle Zahlen und Formeln, auch einzelne Zahlen im Fließtext, werden in LaTeX gesetzt, ebenso griechische Buchstaben oder physikalische Einheiten. Dies gewährleistet ein einheitliches Erscheinungsbild und die korrekte Darstellung von Zahlen und Einheiten.

Für die übersichtliche Gestaltung der Lernmodulseiten wurde mit wiederkehrenden grafischen Elementen gearbeitet, die sich vom Fließtext abheben und optisch gut unterscheidbar sind (vergleiche auch Abbildung 8):

- Mathematische Definitionen, Sätze und Rechenregeln werden durch einen hellgrünen Block und die Überschrift „Definition:“ (bzw. „Satz:“) gekennzeichnet.

- Hinweise und Anmerkungen befinden sich in einem gelben Block mit der Überschrift „Merke:““.

- Beispielrechnungen oder Anwendungsbeispiele werden durch die Überschrift „Beispiel:““(dunkelgraue Überschrift über grauer Linie) vom Text abgehoben. 


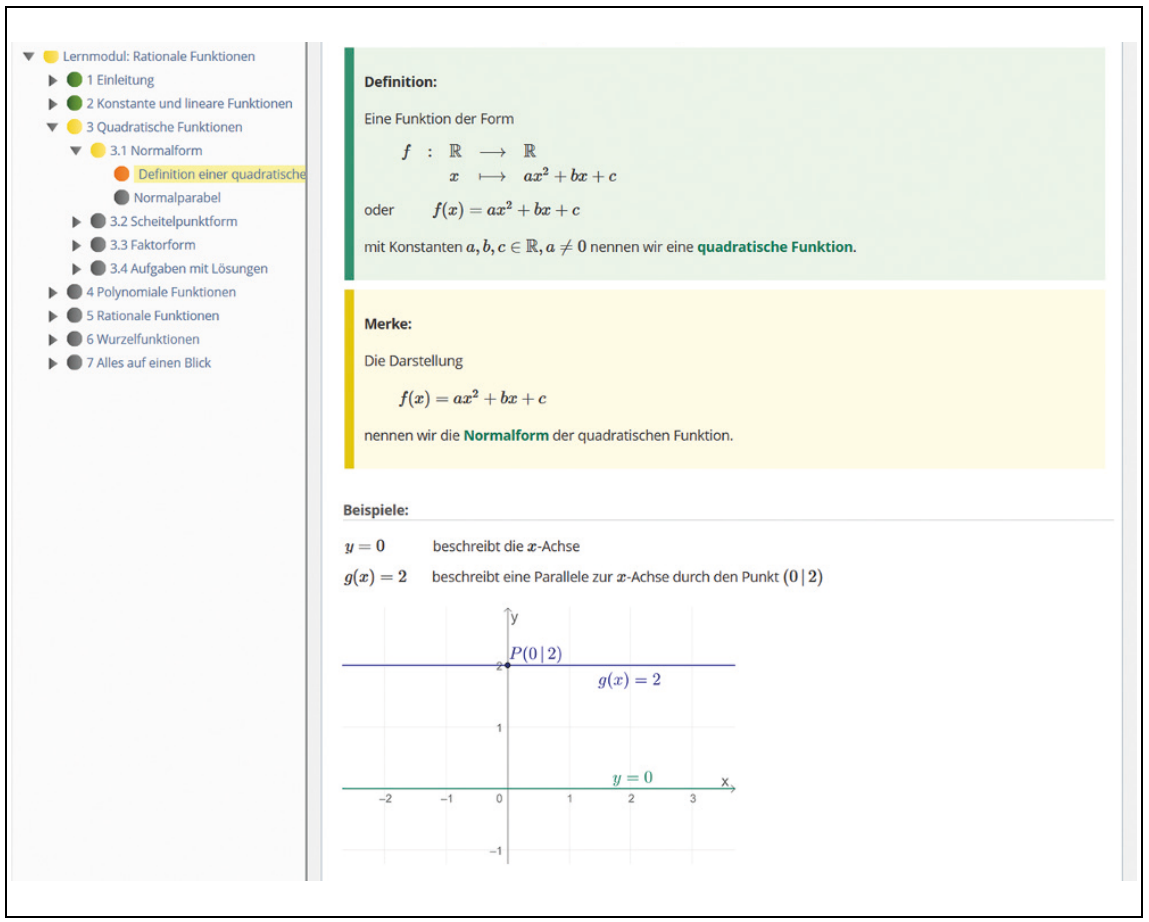

Abbildung 8: Screenshot Lernmodul Rationale Funktionen und Wurzelfunktionen (LoK Funktionen): Inhaltsseite mit Definition, Merke-Block und Beispiel

Zur leichteren Orientierung auf der Lernplattform und zur besseren Wiedererkennung der Inhalte wurden für optes verschiedene Icons entwickelt, beispielsweise für die Themengebiete der mathematischen Kurse oder den Diagnostischen Einstiegstest bzw. Abschlusstest. In den Lernmodulen wird den Lernenden außerdem anhand eines Icons angezeigt, ob sie eine Aufgabe mit oder ohne Taschenrechner bearbeiten sollten (siehe Tabelle 2).

Im Text werden außerdem Begriffe verlinkt - entweder intern auf andere Lernmodulseiten oder innerhalb von ILIAS auf das Glossar oder auf eine externe Website. Für diese drei Fälle wurden unterschiedliche Hervorhebungsfarben definiert (siehe Tabelle 3). 
Tabelle 2: Für optes entwickelte Icons (Design: Carolin Wanner)

\begin{tabular}{|c|c|c|c|c|}
\hline \multicolumn{5}{|c|}{ Einstiegs- / Abschlusstest } \\
\hline Yo & $\begin{array}{l}\text { Diagnostischer } \\
\text { Einstiegstest (A) }\end{array}$ & \multicolumn{3}{|c|}{-® Abschlusstest (E) } \\
\hline \multicolumn{5}{|c|}{ Kurs-Icons } \\
\hline$\Sigma$ & $\begin{array}{l}\text { Mathematische } \\
\text { Grundlagen }\end{array}$ & $\begin{array}{l}+ \\
\end{array}$ & Arithmetik & $\begin{array}{l}\mathbf{E}< \\
\geq\end{array}$ \\
\hline$\sqrt[n]{x}$ & $\begin{array}{l}\text { Potenzen, } \\
\text { Wurzeln, } \\
\text { Logarithmen }\end{array}$ & $f(x)$ & Funktionen & 1 Geometrie \\
\hline & Trigonometrie & & Lineare Algebra & $\begin{array}{l}\lim _{x \rightarrow \infty} \begin{array}{l}\text { Folgen, Grenzwerte } \\
\text { und Stetigkeit }\end{array}\end{array}$ \\
\hline$\frac{d}{d x} \int$ & $\begin{array}{l}\text { Differential und } \\
\text { Integralrechnung }\end{array}$ & $\Leftrightarrow$ & Logik & $\because$ Statistik \\
\hline \multicolumn{5}{|c|}{ Taschenrechner Icon } \\
\hline 㤟 & $\begin{array}{l}\text { Taschenrechner } \\
\text { „erlaubt" }\end{array}$ & $F_{f i}$ & \multicolumn{2}{|c|}{$\begin{array}{l}\text { Ohne Taschenrechner } \\
\text { bearbeiten }\end{array}$} \\
\hline
\end{tabular}

Auch die in optes eingebundenen Grafiken und Animationen orientieren sich an einem Farbschema. Damit die Autor*innen dieser Grafiken eine gewisse Freiheit bei der Gestaltung haben, wurden acht Farbwerte zur Darstellung von Linien, Graphen oder Beschriftungen vorgegeben (siehe Tabelle 3).

Die in diesem Kapitel vorgestellten Gestaltungselemente können nur einen Eindruck vermitteln, wie wichtig die Formulierung und Einhaltung von verbindlichen Gestaltungsregeln für die Usability einer Mathematik-Lernumgebung sind. Dies gilt insbesondere in größeren Projekten wie optes mit mehreren Teams. Damit alle optes-Autor*innen über diese Regeln informiert sind, wurde eine umfangreiche und verbindliche Handreichung erstellt, die auch auf der optes-Plattform einsehbar ist (Derr, Mechelke-Schwede und Weigel 2019). 
Tabelle 3: Farben in optes-Lernmaterialien

\begin{tabular}{|c|c|c|c|c|}
\hline & hex & rgb & $\begin{array}{l}\text { Farbmuster } \\
\text { Linie }\end{array}$ & $\begin{array}{l}\text { Farbmuster } \\
\text { Fläche }\end{array}$ \\
\hline Optes-Logo helles Grün & \#3AAA35 & $58,170,53$ & Text ---+-- & \\
\hline Optes-Logo dunkles Grün & \#2D8E86 & $45,142,134$ & Text ---+-- & \\
\hline \multicolumn{5}{|l|}{ Hervorhebungen im Text } \\
\hline $\begin{array}{l}\text { Link auf Glossarbegriff } \\
\text { (intern) }\end{array}$ & \#007959 & $0,121,89$ & Text ---+-- & \\
\hline $\begin{array}{l}\text { Link auf Seite oder Kapitel } \\
\text { (intern) }\end{array}$ & \#00008B & $0,0,139$ & Text ---+-- & \\
\hline $\begin{array}{l}\text { Link auf externe Seite } \\
\text { (extern) }\end{array}$ & \#660000 & $102,0,0$ & Text ---+-- & \\
\hline $\begin{array}{l}\text { Erste Hervorhebung im Text } \\
\text { Zweite Hervorhebung im }\end{array}$ & \#FF8C00 & $255,140,0$ & Text ---+-- & \\
\hline Text & \#CD853F & $205,133,63$ & Text ---+-- & \\
\hline \multicolumn{5}{|c|}{ Farben in Grafiken und Applets } \\
\hline Farbe 1 & \#007959 & $0,121,89$ & Text ---+-- & \\
\hline Farbe 2 & \#00008B & $0,0,139$ & Text ---+-- & \\
\hline Farbe 3 & \#660000 & $102,0,0$ & Text ---+-- & \\
\hline Farbe 4 & \#FA4D14 & $250,77,20$ & Text ---+-- & \\
\hline Farbe 5 & \#A0229B & $160,34,155$ & Text ---+-- & \\
\hline Farbe 6 & \#73AD54 & $115,173,84$ & Text ---+-- & \\
\hline Farbe 7 & \#64A1EB & $100,161,235$ & Text ---+-- & \\
\hline Farbe 8 & \#В08963 & $176,137,99$ & Text ---+-- & \\
\hline
\end{tabular}

\section{Literatur}

Clark, R. C. \& Mayer, R. E. (2011). E-learning and the science of instruction. Proven guidelines for consumers and designers of multimedia learning (3. Aufl.). San Francisco: Pfeiffer.

Derr, K., Mechelke-Schwede, E. \& Weigel, M. (2019). Handreichung Lernmodule, Tests und Fragenpools. TP 3 Formatives eAssessment \& Propädeutika.

DIN Norm. Ergonomics of human-system interaction - Part 11: Usability: Definitions and concepts. ISO 9241-11:2018.

Duckwitz, A. \& Leuenhagen, M. (2004). Usability und E-Learning. Rezeptionsforschung für die Praxis. In D. Carstensen \& B. Barrios (Hrsg.), Campus 2004. Kommen die digitalen Medien an den Hochschulen in die Jahre? (Medien in der Wissenschaft, Bd. 29, S. 36-45). Münster: Waxmann.

Gizycki, V. von. (2002). Usability - nutzerfreundliches Web-Design. In M. Beier \& V. von Gizycki (Hrsg.), Usability. Nutzerfreundliches Web-Design (S. 1-18). Berlin: Springer.

Granić, A. \& Ćukušić, M. (2011). Usability Testing and Expert Inspections Complemented by Educational Evaluation. A Case Study of an e-Learning Platform. Educational Technology \& Society, 14(2), 107-123. 
Krause, U.-M. \& Stark, R. (2006). Vorwissen aktivieren. In H. Mandl \& H. F. Friedrich (Hrsg.), Handbuch Lernstrategien (S. 38-49). Göttingen: Hogrefe.

Lera, E. de, Almirall, M., Valverde, L. \& Gisbert, M. (2013). Improving User Experience in e-Learning, the Case of the Open University of Catalonia. In A. Marcus (Hrsg.), Design, User Experience, and Usability. Health, Learning, Playing, Cultural, and CrossCultural User Experience (S. 180-188). Berlin: Springer.

Marcus, A. (Hrsg.) (2013). Design, User Experience, and Usability. Health, Learning, Playing, Cultural, and Cross-Cultural User Experience. Berlin: Springer.

Nielsen, J. (1994). Heuristic Evaluation. In J. Nielsen \& R. L. Mack (Hrsg.), Usability Inspection Methods (S. 25-64). New York: Wiley.

Nielsen, J. (2010). College Students on the Web. Verfügbar unter https://www. nngroup.com/articles/college-students-on-the-web/ [12.06.2020].

Reeves, T. C. (1994). Evaluating what really matters in computer-based education. In M. Wild \& D. Kirkpatrick (Hrsg.), Computer education: New Perspectives (S. 219-246). Perth: Mastec.

Scholtz, B., Mahmud, I. \& Ramayah, T. (2016). Does usability matter? An analysis of the impact of usability on technology acceptance in ERP settings. Interdisciplinary Journal of Information, Knowledge, and Management, (11), 309-330.

Winne, P. H. (2004). Students' calibration of knowledge and learning processes: Implications for designing powerful software learning environments. International Journal of Educational Research, 41(6), 466-488.

Open Access Dieses Kapitel wird unter der Creative Commons Namensnennung 4.0 International Lizenz (http://creativecommons.org/licenses/by/4.0/deed. de) veröffentlicht, welche die Nutzung, Vervielfältigung, Bearbeitung, Verbreitung und Wiedergabe in jeglichem Medium und Format erlaubt, sofern Sie den/ die ursprünglichen Autor(en) und die Quelle ordnungsgemäß nennen, einen Link zur Creative Commons Lizenz beifügen und angeben, ob Änderungen vorgenommen wurden.

Die in diesem Kapitel enthaltenen Bilder und sonstiges Drittmaterial unterliegen ebenfalls der genannten Creative Commons Lizenz, sofern sich aus der Abbildungslegende nichts anderes ergibt. Sofern das betreffende Material nicht unter der genannten Creative Commons Lizenz steht und die betreffende Handlung nicht nach gesetzlichen Vorschriften erlaubt ist, ist für die oben aufgeführten Weiterverwendungen des Materials die Einwilligung des jeweiligen Rechteinhabers einzuholen.

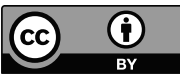

